ORIGINAL ARTICLE

\title{
Continuous end-tidal carbon dioxide monitoring for confirmation of endotracheal tube placement is neither widely available nor consistently applied by emergency physicians
}

\section{N M Delorio}

Objectives: To determine the availability of end-tidal $\mathrm{CO}_{2}$ measurement in confirmation of endotracheal tube placement in the non-arrest patient, and to assess its use in academic and non-academic emergency departments.

Methods: Emergency physicians in the USA were surveyed by mail in the beginning of the year 2000 regarding availability at their institution of both colorimetric/qualitative and quantitative end-tidal $\mathrm{CO}_{2}$ capnography, frequency of use in their own practice, and descriptor of their hospital (academic, community teaching, and community non-teaching). Additionally, data were obtained from the National Emergency Airway Registry 97 series (NEAR) about how many intubations used this method of confirmation. NEAR site coordinators were surveyed as well.

Results: Of 1000 surveys, 550 were returned (55\%). Colorimetric technology existed in $77 \%$ of respondents' hospitals ( $n=421$ ); $25 \%$ of respondents $(n=138$ ) had continuous monitoring capability. Physicians practising at academic hospitals were more likely to have continuous monitoring (36\%; $n=196$ )

Correspondence to: N M Delorio, Oregon Health and Science University, 3181 SW Sam Jackson Park Rd, Portland, OR 97239, USA; deiorion@ohsu.edu

Accepted for publication 9 June 2004 than community teaching institutions $(32 \% ; n=173)$ and non-teaching centres $(18 \% ; n=100)(p<0.001)$. Among physicians who had this technology available, only $14 \%(n=19)$ "always" used it in non-arrest intubations; $57 \%$ "rarely" or "never" employed it $(n=75)$. Among NEAR centres (institutions committed to monitoring current airway practices) only $12 \%$ of $6009(n=716)$ intubations used continuous end-tidal $\mathrm{CO}_{2}$ measurement. Of these practitioners, only $40 \%$ "always" used it $(\mathrm{n}=6 / 15)(83 \%$ response rate $(n=29 / 35))$.

Conclusions: Despite recommendations from national organisations that endorse continuous monitoring of end-tidal $\mathrm{CO}_{2}$ for confirming endotracheal tube placement, it is neither widely available nor consistently applied.
$\mathrm{E}$ ndotracheal intubation is "a potential minefield for disaster". ${ }^{1}$ Errors in its performance can be associated with high morbidity and mortality for the patient, and legal liability for the practitioner. Many methods exist for the verification of successful tube placement in the trachea, including watching the tube pass through the cords, pulse oximetry monitoring, and listening for breath sounds. However, none of these are theoretically as accurate as endtidal carbon dioxide $\left(\mathrm{CO}_{2}\right)$ monitoring in the non-cardiacarrest patient. A recent UK survey of intensivists found that although $80 \%$ of intensive care units have end-tidal $\mathrm{CO}_{2}$ monitoring capability, only $50 \%$ of these use it to confirm correct tube placement. Of these $50 \%$, only approximately a third use it for every intubation. ${ }^{2}$ Recently, the American College of Emergency Physicians (ACEP) joined several other institutions in recommending that this modality be used in confirmation and endorse it as the "most accurate". ${ }^{34}$ However, two things remain unclear: (a) What the availability of this modality is in emergency departments and $(b)$ whether physicians' practice patterns reflect the strong wording of these positions?

The purpose of the present study was to understand more fully if this technology is actually being used in emergency departments.

\section{METHODS: PART I}

\section{Survey design and population}

A single-page survey was developed and validated on a pilot sample of 10 emergency physicians. The survey population consisted of 1000 active emergency physician members of the ACEP. A random sample of active physicians' names and addresses were purchased from the organisation. The survey portions of the study (parts I and II) met the criteria for exemption from the institutional review board requirement for informed consent from participants.

\section{Survey content and administration}

A single mailing was carried out via US Mail. No incentives were offered for responding, though we provided a selfaddressed stamped envelope for returning the completed survey. Participants were asked voluntarily for their help. Questions asked included whether the respondent's primary hospital had colorimetric and/or quantitative end-tidal $\mathrm{CO}_{2}$ measurement available, how often the physician used each of these modalities in confirming non-arrest intubations (always, often, rarely, never), and the type of hospital (academic, community teaching, or community non-teaching) at which the respondent practised. A multiple-choice format was used, and the name of the respondent's hospital was elicited, though in a separate area of the questionnaire. Respondents were notified that this information would be removed before data analysis. The author's email address was provided. See box 1 for the complete survey instrument.

\section{Data analysis}

Percentages were calculated for answers to questions 1, 2, 4, 5, and 8. Multivariate analysis was performed regarding the relation between type of hospital (question 8 ) and answers to 


\section{Box 1: Survey instrument}

1. Is colorimetric ETCO2 measurement (e.g. Easy-Cap) available at your department?

- Yes

- No

2. If so, how often do you use this method to confirm ETT placement in non-arrest intubations?

- Always

- Often

- Rarely

- Never

3. If you have this modality available but choose not to always use it, why not? (May choose more than one)

- Feel other methods (e.g. direct visualization, auscultation of breath sounds) are more effective

- Cost

- Difficulty of use

- Use continuous monitoring instead

- Other

4. Is continuous, quantitative ETCO2 monitoring available in your department?

- Yes

- No

5. If so, how often do you use this method to confirm ETT placement in non-arrest intubations?

- Always

- Often

- Rarely

- Never

6. If you have this modality available but choose not to always use it, why not?

- Feel other methods (e.g. direct visualization, auscultation of breath sounds) are more effective

- Cost

- Difficulty of set-up

- Other

7. If you have both colorimetric AND continuous monitoring available, describe how you use these.

- Only use colorimetric method

- Only use continuous method

- First confirm with color change, then hook up quantitative monitor

- Nurse already has continuous monitor set up so colorimetric version unnecessary

- Other

- Not applicable

8. Please select the best descriptor of your hospital.

- Academic

- Community teaching hospital

- Community non-teaching hospital

ETCO2, end-tidal carbon dioxide; ETT, endotracheal tube

questions 1, 2, 4, and 5 (those assessing availability and use of modalities). Questions 3, 6, and 7 were excluded from the data analysis due to lack of usable data.
METHODS: PART II

Design/survey population, content, and administration

In part II of the study, the National Emergency Airway Registry (NEAR 97) was examined. This is a databank of (at the time) 35 hospitals (box 2) which provided information on all their intubations. Data were collected on how many intubations used either qualitative or quantitative end-tidal $\mathrm{CO}_{2}$ to confirm tube placement in non-arrest patients. This information was obtained by correspondence with the registry coordinators. Next, site coordinators of the 35 hospitals were mailed the survey from part I of the study. The NEAR study was approved by the institutional review board of each participating hospital.

\section{Data analysis}

Surveys in this part of the study were analysed as in part I.

\section{RESULTS}

In part I of the study, 550 of 1000 usable surveys were returned (55\%). Ultimately, all surveys were analysed regardless of whether or not multiple physicians from a hospital responded. A total of $77 \%$ physicians reported that colorimetric/qualitative technology exists at their hospitals $(\mathrm{n}=421)$. However, continuous monitoring was available at only $25 \%(n=138)$ of respondents' sites.

Regarding differences between types of hospital, academic practitioners were more likely to have continuous monitoring available $(36 \% ; n=196)$ than either community teaching physicians $(32 \% ; n=173)$ or non-teaching physicians $(18 \%$; $\mathrm{n}=100)(\mathrm{p}<0.001)$. Among the physicians who had this technology in their emergency departments $(n=138), 14 \%$ "always" used it in non-arrest settings $(\mathrm{n}=19)$, whereas $57 \%$ "rarely" or "never" employed it $(\mathrm{n}=75)$. The availability of qualitative technology varied by respondents' hospital type, with $89 \%$ of academic hospitals, $83 \%$ of community teaching hospitals, and $70 \%$ of non-teaching hospitals having this capability.

Part II of the study provided endotracheal tube placement confirmation data in 6009 of the registry's 6695 non-arrest intubations. Of these, $12 \%(\mathrm{n}=716)$ used quantitative endtidal $\mathrm{CO}_{2}$ assessment, and $60 \%(\mathrm{n}=3608)$ were confirmed with qualitative methods. Thus, at least $28 \%$ of intubations used neither type of device.

Of 35 NEAR site coordinators, 29 responded to the survey $(83 \%)$. In $52 \%(n=15)$ of NEAR hospitals, continuous endtidal $\mathrm{CO}_{2}$ monitoring was available; $86 \%$ of site coordinator respondents $(n=25)$ did practise at a site where colorimetric technology was available. Continuous detection was used "rarely" or "never" by $47 \%(\mathrm{n}=7)$ of responders from centres with this modality; $40 \%$ "always" used it $(\mathrm{n}=6)$. Continuous monitoring was available in 4/6 NEAR community teaching hospitals (67\%), 11/20 academic hospitals $(55 \%)$, and $1 / 3$ community non-teaching hospitals $(p=$ not significant)

\section{DISCUSSION}

Multiple position statements have recently endorsed or mandated the use of end-tidal $\mathrm{CO}_{2}$ detectors in the confirmation of endotracheal tube placement. In March 2001, the Emergency Medicine Journal issued this declaration"

Independent confirmation of correct tube placement by the use of devices that detect end-tidal $\mathrm{CO} 2$ is mandatory for every endotracheal intubation performed in the emergency department and as part of the assessment of all patients who arrive at the emergency department already intubated. 
Box 2 Participating National Emergency Airway Registry 1997 hospitals

- Arizona Heart Hospital, Phoenix, AZ

- Brigham and Women's Hospital, Boston, MA

- Children's Hospital Boston, Boston, MA

- Christiana Care Health Systems, Newark, DE

- Case Western Reserve University/Metrohealth Medical Center, Cleveland, $\mathrm{OH}$

- Dartmouth Hitchcock Medical Center, Lebanon, NH

- Erie County Medical Center, Buffalo, NY

- George Washington University, Washington, DC

- Georgetown University Medical Center, Washington, DC

- Hamilton Health Sciences Corporation, Hamilton, ON

- Highland General Hospital, Oakland, CA

- Our Lady of Lourdes Hospital, Camden, NJ

- Maricopa Medical Center, Phoenix, AZ

- Mount Auburn Hospital, Cambridge, MA

- National University Hospital, Singapore

- New York Presbyterian Hospital, New York, NY

- Oregon Health Sciences University, Portland, OR

- Singapore General Hospital, Singapore

- St. Francis Hospital and Medical Center, Hartford, CT

- St. Luke's-Roosevelt Hospital Center, New York, NY

- St. Paul's Hospital, Vancouver, BC

- Stanford University, Stanford, CA

- Sterling Health Group/Emory Peachtree, Atlanta, GA

- The Cambridge hospital, Cambridge, MA

- University of California Davis Medical Center, Sacramento, CA

- University of California at San Diego Medical Center, San Diego, CA

- University of Arizona, Tucson, AZ

- University of Cincinnati, Cincinnati, $\mathrm{OH}$

- University of Florida Health Science Center, Jacksonville, FL

- University of Louisville, Louisville, KY

- University of North Carolina Hospitals, Chapel Hill, NC

- Vanderbilt University, Nashville, TN

In October 2001, the ACEP published the following recommendations ${ }^{3}{ }^{4}$ :

During intubation, direct visualization of the endotracheal tube passing through the vocal cords into the trachea constitutes firm evidence of correct tube placement, but should be verified with additional techniques.

End-tidal $\mathrm{CO}_{2}$ detection, either qualitative, quantitative, or continuous, is the most accurate and easily available method to monitor correct endotracheal tube position in patients who have adequate tissue perfusion.

The National Association of EMS Physicians (NAEMSP) echoes this sentiment in its position statement ${ }^{6}$ :

In the patient with a perfusing rhythm, end-tidal $\mathrm{CO}_{2}$ detection is the best method for verification.
End-tidal $\mathrm{CO}_{2}$ detection has also appeared in the 2002 American Heart Association (AHA) protocols for Advanced Cardiac Life Support (ACLS $)^{7}$ :

Expired $\mathrm{CO}_{2}$ detectors are very reliable in patients with perfusing rhythms and are recommended to confirm tube position in these patients (Class Ila).

Finally, the Association of Anaesthetists of Great Britain and Ireland and the American Society for Anesthesiologists (ASA) embrace the use of end-tidal $\mathrm{CO}_{2}$ detection in their guidelines:

[Capnography is] essential to the safe conduct of anaesthesia $^{8}$

Continual monitoring for the presence of expired carbon dioxide shall be performed unless invalidated by the nature of the patient, procedure or equipment ${ }^{9}$

The present study found that even in the light of strong position statements supporting the use of end-tidal $\mathrm{CO}_{2}$ monitoring in the confirmation of intubations by many professional societies in emergency medicine, anaesthesia, and prehospital care, emergency departments are not universally endorsing this modality. The vast majority of physicians did not have quantitative monitoring at their disposal at the time of the study. Though many more do have the use of qualitative methods such as colorimetric devices, even this number is not $100 \%$. The type of hospital influences the likelihood of available technology. Even the NEAR sites, ostensibly centres committed to monitoring and researching airway practices, report similarly low rates of availability and use of this modality.

More disheartening is the fact that physicians have not embraced this practice even if it is available in their practice settings. Though not officially analysed in the study results because of a lack of valid responses, the survey instrument did include a space for respondents to write in reasons they might have felt such monitoring was not necessary. Reasons cited for this included the belief that other methods, such as colorimetric monitoring, pulse oximetry, visualising tube condensation, or chest radiography were just as reliable, and a perceived difficulty of set-up. Unfortunately, literature does not support the adequacy of alternative methods. In the presence of a continuous waveform, quantitative assessment of end-tidal $\mathrm{CO}_{2}$ should be the gold standard for intubation confirmation. Pulse oximetry may remain in the normal range for up to five minutes after the cessation of lung ventilation. ${ }^{6}$ Colorimetric or qualitative assays have reported sensitivities of as low as $72 \%$ in one prospective study of 106 patients for detecting esophageal intubation. ${ }^{10}$ Another study found a sensitivity of $98 \%$ but a specificity of only $78 \% .{ }^{11}$ A recent Best Evidence Topic Report in this journal concluded that ${ }^{12}$

[t] ]he colourimetric $\mathrm{CO}_{2}$ detector is as accurate as $I R$ capnography at detecting tracheal intubation, but is potentially less accurate at detecting oesophageal intubation.

Esophageal detector devices have a reported accuracy of as low as $50 \%{ }^{13}$ Clearly, these alternative methods are not enough, though the study respondents may not be aware of these numbers.

\section{Limitations}

This study does have limitations that open the way for future research in this area. The response rate of $55 \%$, although good 
for a survey, does leave the practices of almost one half of the target population unknowable. Data from the study population of ACEP members may not be generalisable to emergency physicians as a whole, especially those in other countries. The nature of a survey study makes the possibility of reporting bias inevitable. Finally, capnography is a new, rapidly expanding technology that may be more available today than at the time of the study. Still, it would seem that those who responded to the survey would be over-representative of physicians who use this, suggesting that real life numbers would actually be even lower.

\section{CONCLUSIONS}

This study shows that despite strong calls for the practice of confirming endotracheal tube placement with end-tidal $\mathrm{CO}_{2}$ measurement, our specialty's practitioners are not in compliance. Physicians and operational managers in emergency departments need to be further educated so that this technology becomes more widely available and more consistently used.

Competing interests: none declared

\section{REFERENCES}

1 Clyburn P, Rosen M. Accidental oesophageal intubation. Br J Anaesth 1994;73:55-63.
2 Kannan S, Manji M. Survey of use of end-tidal carbon dioxide for confirming tracheal tube placement in intensive care units in the UK. Anaesthesia 2003;5:476-9.

3 Verification of endotracheal tube placement: policy statement. American College of Emergency Physicians. www.acep.org/1,4923,0.html (accessed 16 February 2004).

4 Verification of endotracheal intubation: policy resource and education papers. American College of Emergency Physicians. www.acep.org/ 1,4924,0.html (accessed 16 February 2004).

5 Position statement number 1 . Confirmation of endotracheal tube placement with end tidal $\mathrm{CO}_{2}$ detection. Emerg Med J 2001;18:329, review March, 2003.

6 O'Connor RE, Swor RA. Verification of endotracheal tube placement following intubation. National Association of EMS Physicians Standards and Clinical Practice Committee. Prehosp Emerg Care 1999;3:248-50.

7 American Heart Association. Guidelines 2000 for cardiopulmonary resuscitation and emergency cardiovascular care. Circulation 2000;102(8 suppl):186-189.

8 Recommendations for Standards of Monitoring During Anaesthesia and Recovery. 3rd edition, December 2000. The Association of Anaesthetists of Great Britain and Ireland. www.aagbi.org/guidelines.html (accessed 9 May 2004).

9 The American Society of Anesthesiologists. Standards for Basic Anesthetic Monitoring. Approved by House of Delegates, October 1986, amended 1998. http://www.asahq.org/publicationsAndServices/standards/ 02.pdf\#2 (accessed 16 February 2004)

10 MacLeod BA, Heller MB, Gerard J, et al. Verification of endotracheal tube placement with colorimetric end-tidal $\mathrm{CO}_{2}$ detection. Ann Emerg Med 1991;20:267-70.

11 Li J. A prospective multicenter trial testing the Scoti device for confirmation of endotracheal tube placement. J Emerg Med 2001;20:231-9.

12 Hogg K, Teece S. Colourimetric $\mathrm{CO}_{2}$ detector compared with capnography for confirming ET tube placement. Emerg Med J 2003;20:265-6.

13 Pelucio M, Halligan L, Dhindsa $\mathrm{H}$. Out-of-hospital experience with the syringe esophageal detector device. Acad Emerg Med 1997;4:563-8. 